\title{
Methods for separation of deuterons produced in the medium and in jets in high-energy collisions
}

\author{
Natasha Sharma, ${ }^{1}$ Tony Perez, ${ }^{2}$ Andy Castro, ${ }^{2}$ Lokesh Kumar, ${ }^{1}$ and Christine Nattrass ${ }^{2}$ \\ ${ }^{1}$ Department of Physics, Panjab University, Chandigarh 160014, India \\ ${ }^{2}$ Department of Physics and Astronomy, University of Tennessee, Knoxville, Tennessee 37996, USA
}

(Received 7 March 2018; published 30 July 2018)

\begin{abstract}
Coalescence has long been used to describe the production of light (anti)nuclei in heavy-ion collisions. The same underlying mechanism may also exist in jets when a proton and a neutron are close enough in phase space to form a deuteron. We model deuteron production in jets by applying an afterburner, which uses a coalescence mechanism to form deuterons from protons and neutrons produced in PYTHIA for $p+p$ collisions at a center of mass energy $\sqrt{s}=7 \mathrm{TeV}$. PYTHIA provides a reasonable description of the proton spectra and the shape of the deuteron spectrum predicted by the afterburner is in agreement with the data. We show that the rise in the coalescence parameter $B_{2}$ with momentum observed in data is consistent with coalescence in jets. We show that dihadron correlations can be used to separate the contributions from the jet and the underlying event. This model predicts that the conditional coalescence parameter in the jetlike correlation should be independent of the trigger momentum.
\end{abstract}

DOI: 10.1103/PhysRevC.98.014914

\section{INTRODUCTION}

The production of light (anti)nuclei is of interest in highenergy particle collisions because of the insight that these measurements can provide into particle production mechanisms [1-15]. Since the binding energies of light (anti)nuclei are on the order of a few $\mathrm{MeV}$, they may be formed via coalescence of (anti)nucleons in the later stages of evolution of the system $[16,17]$. On the other hand, the description of light (anti)nuclei yields by thermal models might suggest their thermal production [15]. The recent results from the STAR experiment on the coefficient of the second term of the Fourier decomposition of the azimuthal anisotropy, $v_{2}$, as a function of transverse momentum of various nuclei show scaling with the number of constituent nucleons [18]. This behavior is expected if light nuclei are formed by the coalescence of nucleons.

In the coalescence approach, the probability of deuteron formation is related to the local density of constituent nucleons as well as their velocities $[16,19]$. The invariant yields of light nuclei can be related to the yields of constituent nucleons by

$$
\begin{aligned}
E_{A} \frac{d^{3} N_{A}}{d^{3} p_{A}} & =B_{A}\left(E_{p} \frac{d^{3} N_{p}}{d^{3} p_{p}}\right)^{Z}\left(E_{n} \frac{d^{3} N_{n}}{d^{3} p_{n}}\right)^{A-Z} \\
& \approx B_{A}\left(E_{p} \frac{d^{3} N_{p}}{d^{3} p_{p}}\right)^{A},
\end{aligned}
$$

where $N_{A}, N_{p}$, and $N_{n}$ represent the yields of a given nucleus, constituent protons, and constituent neutrons, respectively, and

Published by the American Physical Society under the terms of the Creative Commons Attribution 4.0 International license. Further distribution of this work must maintain attribution to the author(s) and the published article's title, journal citation, and DOI. Funded by $S C O A P^{3}$.
$p_{A}, p_{p}$, and $p_{n}$ are their momenta such that $p_{p}=p_{n}=\frac{p_{A}}{A}$. $A$ and $Z$ are the atomic mass number and atomic number, respectively. The coalescence parameter $B_{A}$ reflects the probability of nucleon coalescence. Since the coalescence is expected to happen at a later stage of evolution of the system, the coalescence parameter $B_{A}$ can provide information on the freeze-out correlation volume [16], the effective volume of the nuclear matter at freeze out, i.e., $B_{A} \propto V_{\mathrm{eff}}^{1-A}$. The corresponding coalescence parameter for deuteron production is $B_{2}$ with $A=2$. The $B_{2}$ in heavy-ion collisions decreases with increasing collision energy, consistent with increasing source volume $[15,20]$. Thus, $B_{2}$ measurements are also related to the homogeneity volume from HBT $[21,22]$.

Deuteron production has been measured recently in $p p$ collisions $[15,23]$, an interesting data set to study production through coalescence. Measurements of antinuclei in $p p$ collisions are helpful in searches for dark matter [24-26]. The ALICE experiment has measured the multiplicity dependence of the $d / p$ ratio in $p p, p-\mathrm{Pb}$, and $\mathrm{Pb}-\mathrm{Pb}$ collisions [27]. The ratio increases with multiplicity from $p p$ to $p-\mathrm{Pb}$ collisions, approaching the level in $\mathrm{Pb}-\mathrm{Pb}$ collisions where it remains constant as a function of multiplicity. The full range of data could not be fully explained by either a coalescence or a thermal model [27].

In this paper we study the coalescence mechanism for deuteron production in $p p$ collisions in the Monte Carlo event generator PYTHIA [28]. The PYTHIA model does not generate deuterons so we use the coalescence mechanism as an afterburner for their production from the protons and neutrons generated in PYTHIA [29].

Section II describes the afterburner and Sec. III A compares the proton and deuteron spectra in this model to data, demonstrating that this approach generally agrees with the data. We propose the use of dihadron correlations for separating the production of deuterons in jets and in the bulk in Sec. IIIB. 
This would allow a data-driven approach to test the production mechanisms. We summarize in Sec. IV.

\section{PYTHIA WITH COALESCENCE AFTERBURNER}

PYTHIA is a Monte Carlo model that can simulate $p+p$ collisions [28] and has been tuned to several measurements so that it provides a reasonable description of the data [30]. We use the PYTHIA [28] Perugia 2011 tune [30] for the coalescence afterburner for deuteron production. A total of $1.1 \times 10^{9} p+p$ collisions, including diffractive events, were simulated and unstable particles were forced to decay. The event selection is done by setting the PYTHIA parameters $\operatorname{MSEL}=0, \operatorname{MSUB}(92)=0, \operatorname{MSUB}(93)=0, \operatorname{MSUB}(94)=0$, and $\operatorname{MSUB}(95)=0$. Unstable particles are decayed by setting $\operatorname{MSTJ}(22)=2$ and leaving the default $\operatorname{PARJ}(71)=10 \mathrm{~mm}$ so that particles with a proper lifetime less than $10 \mathrm{~mm}$ decay. PYTHIA includes multiparton interactions in order to describe the production of low and intermediate particle production. It includes the production of jets, minijets, and some resonances but does not include correlations from mechanisms such as hydrodynamical flow. At high momenta, particle production in PYTHIA is dominated by jets. It therefore can describe jet production. Since PYTHIA uses the Lund string model for parton fragmentation and has no mechanism for the production of deuterons, it does not predict the production of deuterons in $p+p$ collisions. Hence, deuterons are not formed by default in PYTHIA and we require an afterburner to coalesce protons and neutrons into deuterons.

The coalescence of protons and neutrons close in phase space has been used to describe the production of deuterons in nuclear collisions $[16,17,19]$. We use an afterburner for the production of deuterons as described in Ref. [29]. In this work, the Wigner phase-space density for deuterons is obtained from the Hulthén wave function, which is expressed in terms of the sum of 15 Gaussian wave functions

$$
\phi(r)=\sum_{i=1}^{15} c_{i}\left(\frac{2 w_{i}}{\pi}\right)^{3 / 4} \exp \left(-w_{i} r^{2}\right),
$$

where the coefficient $c_{i}$ and the width parameter $w_{i}$ are determined by least-squares fit, and are given in Table I. It is observed that the sum of 15 Gaussian wave functions reproduced the exact Hulthén wave function both in coordinate and momentum spaces. The deuteron wave function $\rho_{d}^{W}(\vec{r}, \vec{k})$ can then be described analytically by the Wigner phase-space density

$$
\begin{aligned}
\rho_{d}^{W}(\vec{r}, \vec{k})= & 8 \sum_{i=1}^{15} c_{i}^{2} e^{-2 w_{i} r^{2}-k^{2} / 2 w_{i}} \\
& +16 \sum_{i>j}^{15} c_{i} c_{j}\left(\frac{4 w_{i} w_{j}}{\left(w_{i}+w_{j}\right)^{2}}\right)^{3 / 4} \\
& \times e^{\frac{-4 w_{i} w_{j} r^{2}-k^{2}}{w_{i}+w_{j}}} \cos \left(2 \frac{w_{i}-w_{j}}{w_{i}+w_{j}} \vec{r} \cdot \vec{k}\right),
\end{aligned}
$$

where $\vec{r}$ is the relative position of the proton and neutron and $\vec{k}$ is their relative momentum. We evaluate Eq. (3) for the positions
TABLE I. Coefficients describing the Wigner function in Eq. (3).

\begin{tabular}{lcc}
\hline \hline$i$ & $c_{i}$ & $w_{i}\left(1 / \mathrm{fm}^{2}\right)$ \\
\hline 1 & $3.49665 \times 10^{-1}$ & $1.57957 \times 10^{-2}$ \\
2 & $1.85419 \times 10^{-1}$ & $3.94293 \times 10^{-2}$ \\
3 & $1.72279 \times 10^{-1}$ & $8.99793 \times 10^{-2}$ \\
4 & $4.62152 \times 10^{-2}$ & $9.75943 \times 10^{-2}$ \\
5 & $1.49458 \times 10^{-1}$ & $1.80117 \times 10^{-1}$ \\
6 & $7.74205 \times 10^{-2}$ & $1.93353 \times 10^{-1}$ \\
7 & $1.48268 \times 10^{-4}$ & $1.99811 \times 10^{-1}$ \\
8 & $7.35549 \times 10^{-3}$ & $2.17921 \times 10^{-1}$ \\
9 & $4.89047 \times 10^{-2}$ & $2.89902 \times 10^{-1}$ \\
10 & $4.19816 \times 10^{-2}$ & $4.70739 \times 10^{-1}$ \\
11 & $1.72670 \times 10^{-2}$ & $4.89604 \times 10^{-1}$ \\
12 & $1.06294 \times 10^{-1}$ & $9.27621 \times 10^{-1}$ \\
13 & $2.51462 \times 10^{-4}$ & 1.98822 \\
14 & $3.22947 \times 10^{-2}$ & 2.59243 \\
15 & $1.15826 \times 10^{-2}$ & $1.44639 \times 10$ \\
\hline \hline
\end{tabular}

and momenta of final-state protons and neutrons in PYTHIA. Figure 1 shows Eq. (3) as a function of the relative positions and momenta of protons and neutrons. It shows that the probability of deuteron formation through the coalescence of protons and neutrons is higher if they are closer in momentum and coordinate space. The probability decreases with increasing relative position and momentum. Using protons and neutrons produced in PYTHIA and the afterburner described above, we obtain the deuterons from coalescence of protons and neutrons.

The number of deuterons is likely overestimated because PYTHIA assigns the primary vertex as the origin of primary particles. This is a reasonable estimate for many purposes, but may overestimate spatial correlations between protons and neutrons. We nevertheless expect that this method can describe the qualitative behavior of deuteron production in experimental data and allow tests of experimental techniques for investigating deuteron production.

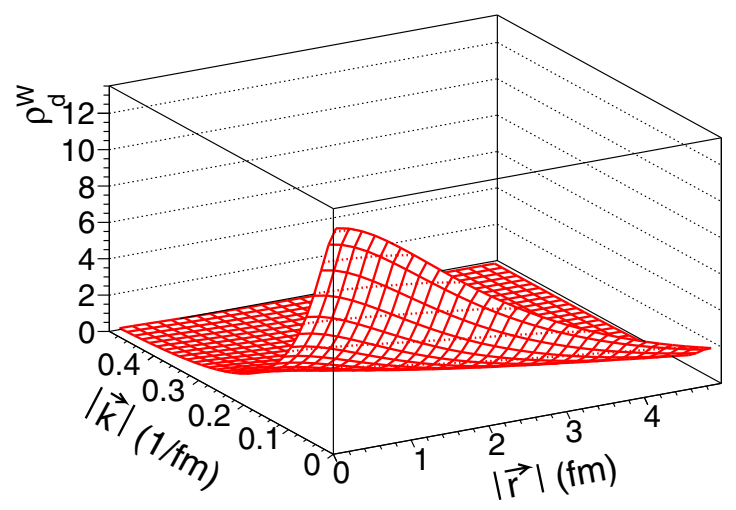

FIG. 1. The deuteron wave function $\rho_{d}^{W}$ defined in Eq. (3) as a function of magnitude of relative position $\vec{r}$ and relative momentum $\vec{k}$ of protons and neutrons for $\vec{r} \cdot \vec{k}=0$. 


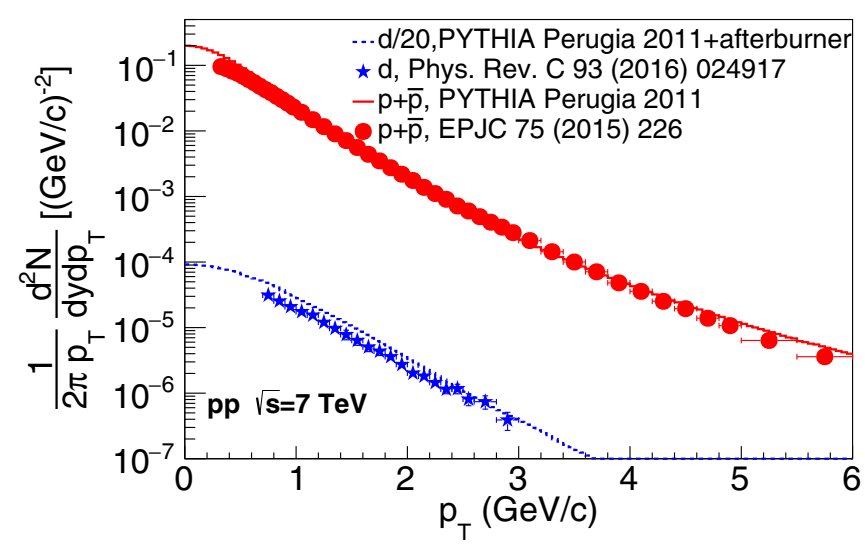

FIG. 2. Proton spectra from [31] and PYTHIA [28] Perugia 2011 tune [30] and deuteron spectra from [15] and PYTHIA Perugia 2011 with the coalescence afterburner described in the text in $p+p$ collisions at $\sqrt{s}=7 \mathrm{TeV}$.

\section{RESULTS}

\section{A. Spectra}

Figure 2 shows the deuteron spectrum from PYTHIA with the coalescence afterburner compared to experimental measurements $[15,23]$. The deuteron spectrum from PYTHIA is scaled by a factor of $1 / 20$. As explained in Sec. II, PYTHIA likely overestimates spatial correlations between protons and neutrons, leading to an overestimate of the number of deuterons produced. The deuteron spectrum from the model has a similar shape to that observed in data. To see how the proton spectrum from PYTHIA compares to the data, we also plot the $p+\bar{p}$ spectrum from PYTHIA with the same from data [31]. PYTHIA gives the correct shape for both protons and deuterons.

Figure 3 shows $B_{2}$ calculated using Eq. (1) from proton [31] and deuteron [15] spectra and from the PYTHIA [28] Perugia 2011 tune [30] with the coalescence afterburner in $p+p$ collisions at $\sqrt{s}=7 \mathrm{TeV}$. The $B_{2}$ from PYTHIA+afterburner overestimates deuterons and has been scaled by $1 / 20$. The

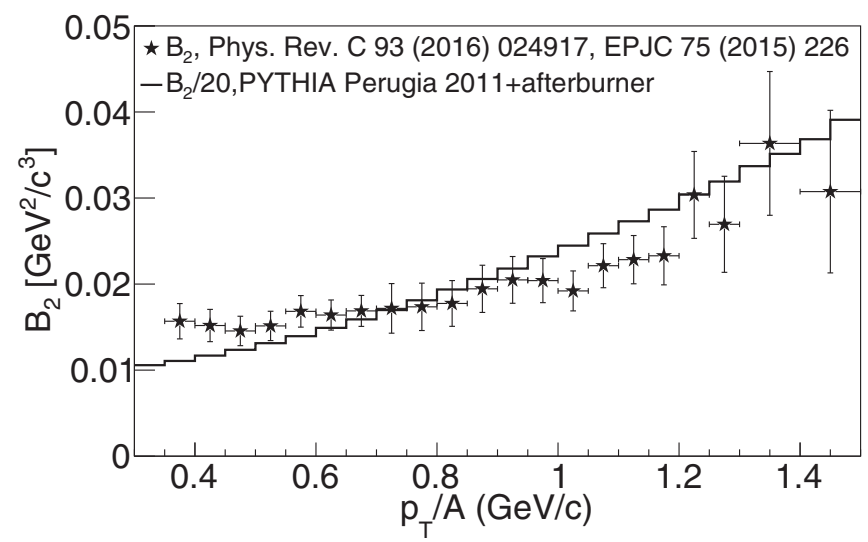

FIG. 3. $B_{2}$ calculated from proton [31] and deuteron [15] spectra and from PYTHIA [28] Perugia 2011 tune [30] with the coalescence afterburner described in the text in $p+p$ collisions at $\sqrt{s}=7 \mathrm{TeV}$.
$B_{2}$ from data and PYTHIA+afterburner show similar behavior, increasing as a function of $p_{\mathrm{T}}$ with similar slopes.

In heavy-ion collisions, $B_{2}$ also shows an increase with increasing $p_{\mathrm{T}}$ [15]. It is expected that $B_{2}$ as a function of $p_{\mathrm{T}}$ would be flat if the deuterons are formed via simple coalescence [15]. The rise in $B_{2}$ as a function of $p_{\mathrm{T}}$ in data is suggested to be due to flow [15] and/or hard scattering [23,32]. The PYTHIA model does not include flow so the rise in $B_{2}$ as a function of $p_{\mathrm{T}}$ in the PYTHIA+afterburner model can be attributed to hard scattering. Moreover, particle production at high momenta in PYTHIA is dominated by jets and minijets. The contribution to deuteron production through coalescence in jets can be studied in jets and the contribution from soft processes can be studied in the underlying event (UE). It is therefore interesting to study the behavior of $B_{2}$ versus $p_{\mathrm{T}}$ for jets and the underlying event separately.

\section{B. Dihadron correlations}

We propose disentangling the contributions from the underlying event and in jets using dihadron correlations, which are frequently used to measure the production of jets in heavy-ion collisions without the need for full jet reconstruction and allow separation between contributions from jets and from flow.

A trigger particle is selected by its high momentum, $p_{T}^{\mathrm{t}}$, and the distribution of particles is measured in azimuth $\Delta \phi=$ $\phi^{t}-\phi^{a}$ and pseudorapidity $\Delta \eta=\eta^{t}-\eta^{a}$ relative to that trigger particle. Here the superscripts $t$ and $a$ are used for the trigger and associated particles, respectively. We restrict reconstructed particles to $|\eta|<0.9$, a range accessible by the ALICE experiment, resulting in a trivial acceptance effect. We correct for this by dividing by $a(\Delta \eta)=1-\frac{1}{1.8} \Delta \eta$. A sample correlation is shown in Fig. 4 for charged hadron $\left(\pi^{ \pm}, K^{ \pm}, p, \bar{p}\right)$ trigger particles with charged hadron associated particles, for charged hadron trigger particles with associated protons, and for charged hadron trigger particles with associated deuterons. A clear peak is seen near $\Delta \phi \approx 0$ and $\Delta \eta \approx 0$, referred to as the near side. This peak is narrow in both $\Delta \phi$ and $\Delta \eta$ and contains particles from the same jet as the trigger particle. There is an additional peak from the partner jet at approximately $\Delta \phi=\pi$, called the away side. This peak is narrow in azimuth but broad in pseudorapidity due to the difference between the center of momentum frame of the hard scattered partons and the incoming protons.

Even in PYTHIA, there is some contribution from the underlying event. Dihadron correlations contain contributions where both the trigger and associated particles are from the same jet $(J-J)$, where the trigger particle is from a jet but the associated particle is not from the same jet $(J-B)$, and where neither the trigger or associated particles are from a jet $(B-B)$. At sufficiently high momenta, contributions from $B-B$ to the correlation function are negligible in $p+p$ collisions. In PYTHIA contributions from $J-B$ can include either associated particles from the underlying event or associated particles produced by a different hard scattering from the trigger particle. Our goal here is to clearly identify deuteron production in jets, $J-J$, requiring a background subtraction.

There are several approaches to background subtraction in dihadron correlations [33]. For simplicity, we focus on the near 

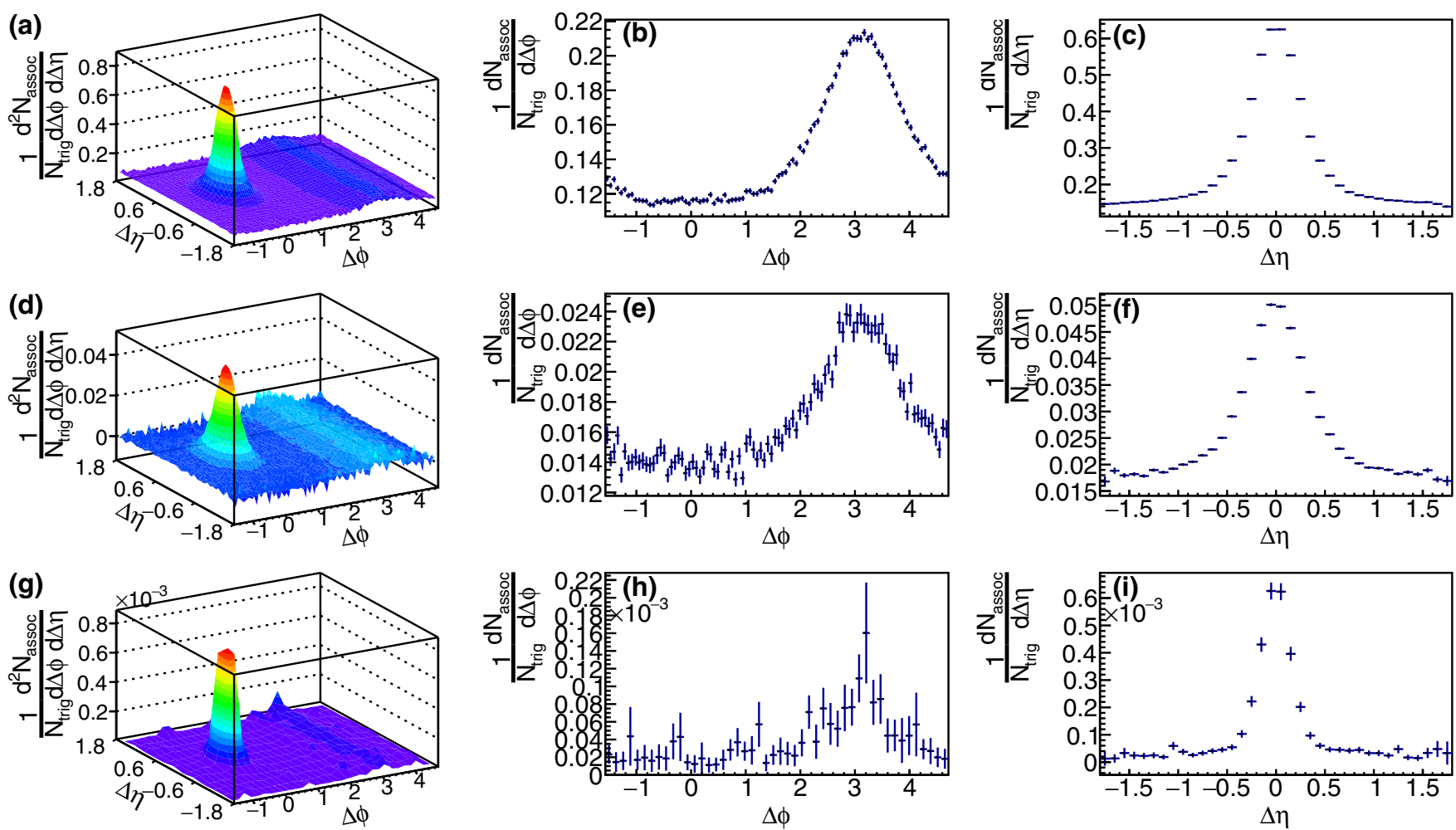

FIG. 4. Dihadron correlations for trigger momenta $5<p_{T}^{\mathrm{t}}<7 \mathrm{GeV} / c$ within pseudorapidities $|\eta|<0.9$ and associated particles within $|\eta|<0.9$ with momenta $2.0<p_{T}^{\mathrm{a}}<3.0 \mathrm{GeV} / c$ in $p+p$ collisions at $\sqrt{s}=7 \mathrm{TeV}$ in PYTHIA [28]. The signal is normalized by the number of trigger particles and corrected for the acceptance as described in the text. Correlation function in $\Delta \phi$ and $\Delta \eta$ is shown for associated charged hadrons (a), associated protons (d), and associated deuterons (g). Correlation function in $\Delta \phi$ for $1.0<|\Delta \eta|<1.8$ is shown for associated charged hadrons (b), associated protons (e), and associated deuterons (h). Correlation function in $\Delta \eta$ for $-\frac{\pi}{2}<|\Delta \phi|<\frac{\pi}{2}$ is shown for associated charged hadrons (c), associated protons (f), and associated deuterons (i).

side. Figure 4 shows the correlation function in $\Delta \phi$ for $1.0<$ $|\Delta \eta|<1.8$ for each combination of trigger and associated particles, demonstrating that contributions of jetlike correlations on the near side to the correlation function are negligible in this range. The correlation function in $\Delta \eta$ on the near side, $-\frac{\pi}{2}<|\Delta \phi|<\frac{\pi}{2}$, is also shown. We estimate the background by fitting this correlation function with a constant over the range $1.0<|\Delta \eta|<1.8$. This approach works in heavy-ion collisions for subtracting the flow-modulated background as well [34-36].

The conditional yield can be calculated as

$$
Y_{\text {trig }}=\frac{1}{N_{\text {trig }}} \int_{-1.8}^{1.8} \int_{-\frac{\pi}{2}}^{\frac{\pi}{2}} \frac{d^{2} N_{\text {assoc }}}{d \Delta \eta d \Delta \phi} d \Delta \phi d \Delta \eta
$$

either for the $J-J$ signal or for the background and the conditional associated spectrum $\frac{1}{2 \pi p_{T}} \frac{d Y_{\text {trig }}}{d p_{T}}$ can be calculated. Equation (4) gives the yield normalized per trigger particle, the conventional normalization for dihadron correlations. In the case when the number of trigger particles that are not from hard processes is negligible, the yield is then the number of associated particles per jet. Yields normalized by the number of trigger particles should be comparable for different systems. When the yield is normalized by the number of events, $Y_{\mathrm{eve}}$, it is a measure of the number of particles produced by jets per event, which can then be compared to the inclusive particle spectra.

Figure 5 shows the conditional spectra for $|\Delta \eta|<1.8$ and $|\Delta \phi|<\frac{\pi}{2}$ for both the jet signal and the underlying event

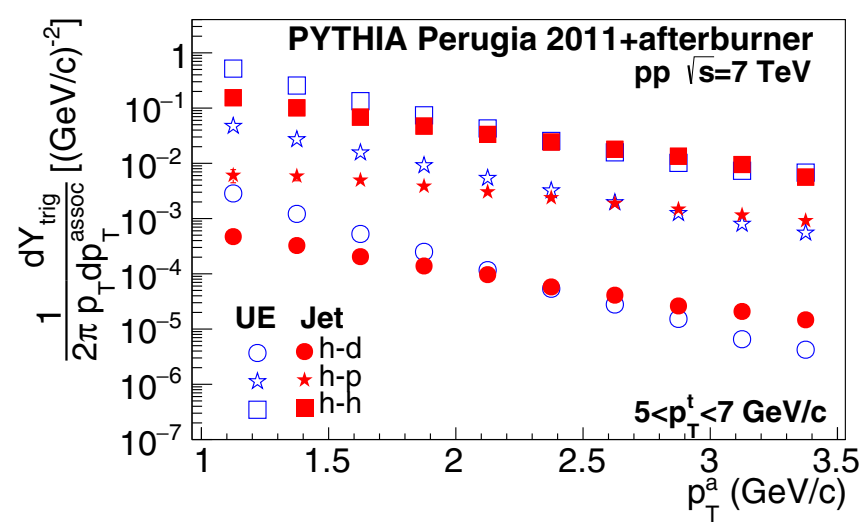

FIG. 5. Conditional spectra $\frac{1}{2 \pi p_{T}} \frac{d Y_{\text {trig }}}{d p_{T}}$ for $|\Delta \eta|<1.8$ and $|\Delta \phi|<$ $\frac{\pi}{2}$ as a function of $p_{T}^{\mathrm{a}}$ for associated charged hadrons (h-h), associated protons (h-p), and associated deuterons (h-d) for $5<p_{T}^{\mathrm{t}}<7 \mathrm{GeV} / c$ within pseudorapidities $|\eta|<0.9$ and associated particles within $|\eta|<0.9$ in $p+p$ collisions at $\sqrt{s}=7 \mathrm{TeV}$ in PYTHIA [28]. The solid symbols denote the jetlike correlation while the open symbols represent the underlying event (UE). 


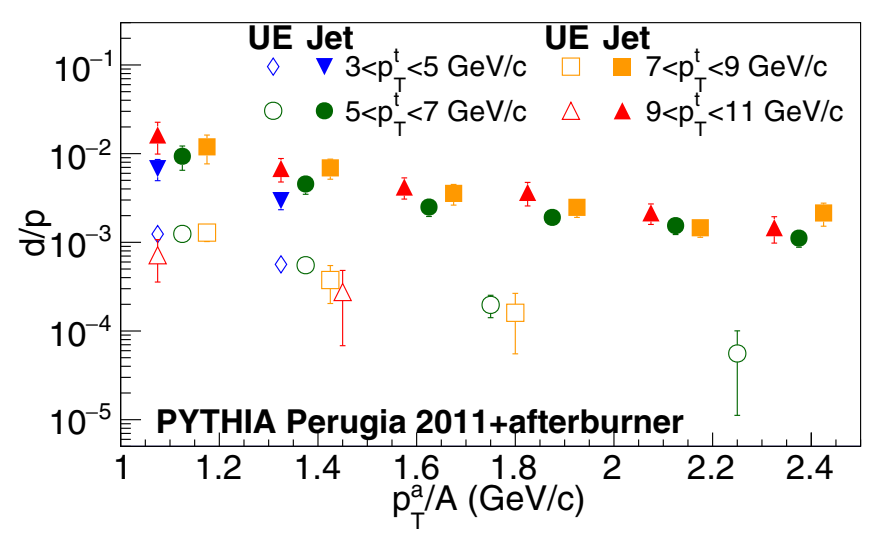

FIG. 6. Ratio of deuteron to proton yields as a function of $p_{T}^{a} / A$ calculated from the conditional spectra for $3<p_{T}^{\mathrm{t}}<5 \mathrm{GeV} / c, 5$ $<p_{T}^{\mathrm{t}}<7 \mathrm{GeV} / c, 7<p_{T}^{\mathrm{t}}<9 \mathrm{GeV} / c$, and $9<p_{T}^{\mathrm{t}}<11 \mathrm{GeV} / c$ for both the jetlike correlation and the underlying event. Points have been displaced for visibility. The solid symbols denote the jetlike correlation while the open symbols represent the underlying event (UE).

background as a function of $p_{T}^{\mathrm{a}}$ for associated charged hadrons (h-h), associated protons (h-p), and associated deuterons (h-d) for $5<p_{T}^{\mathrm{t}}<7 \mathrm{GeV} / c$. The conditional spectra are highest for unidentified hadrons and lowest for deuterons for both the jetlike correlation and the underlying event, reflecting differences in the inclusive production of these particles. For each type of associated particle, the conditional yield is higher for the underlying event than the jetlike correlation at lower $p_{T}^{\mathrm{a}}$ and vice versa for higher $p_{T}^{\mathrm{a}}$. This shows that the jetlike contribution becomes dominant with increasing momentum, which is clearly visible for h-d correlations.

Figure 6 shows the ratio of deuteron to proton yields plotted as a function of $p_{T}^{a} / A$ where $A$ is the mass number. The ratio is calculated from the conditional spectra using the following equation

$$
\frac{d}{p}=\frac{\frac{1}{2 \pi\left(p_{T}^{d} / 2\right)} \frac{d Y_{\text {trig }}^{d}}{d p_{T}^{d} / 2}}{\frac{1}{2 \pi p_{T}^{p}} \frac{d Y_{\text {trig }}^{p}}{d p_{T}^{p}}}
$$

for $3<p_{T}^{\mathrm{t}}<5 \mathrm{GeV} / c, 5<p_{T}^{\mathrm{t}}<7 \mathrm{GeV} / c, 7<p_{T}^{\mathrm{t}}<9$ $\mathrm{GeV} / c$, and $9<p_{T}^{\mathrm{t}}<11 \mathrm{GeV} / c$ for both the jetlike correlation and the underlying event. The ratio is similar for different trigger $p_{\mathrm{T}}$ for both the underlying event and jetlike correlations. The $d / p$ ratio is higher for the jetlike correlation than for the underlying event and decreases with increasing $p_{T}^{a} / A$, however, the ratio decreases faster for the underlying event with increasing $p_{T}^{a} / A$. This suggests that the probability of deuteron production through coalescence is higher in the jetlike correlation than in the underlying event.

An analog to $B_{2}$ can be calculated from these conditional spectra

$$
B_{2}^{\text {trig }}=\frac{\frac{1}{2 \pi\left(p_{T}^{d} / 2\right)} \frac{d Y_{\text {trig }}^{d}}{d p_{T}^{d} / 2}}{\left(\frac{1}{2 \pi p_{T}^{p}} \frac{d Y_{\text {trig }}^{p}}{d p_{T}^{p}}\right)^{2}}
$$

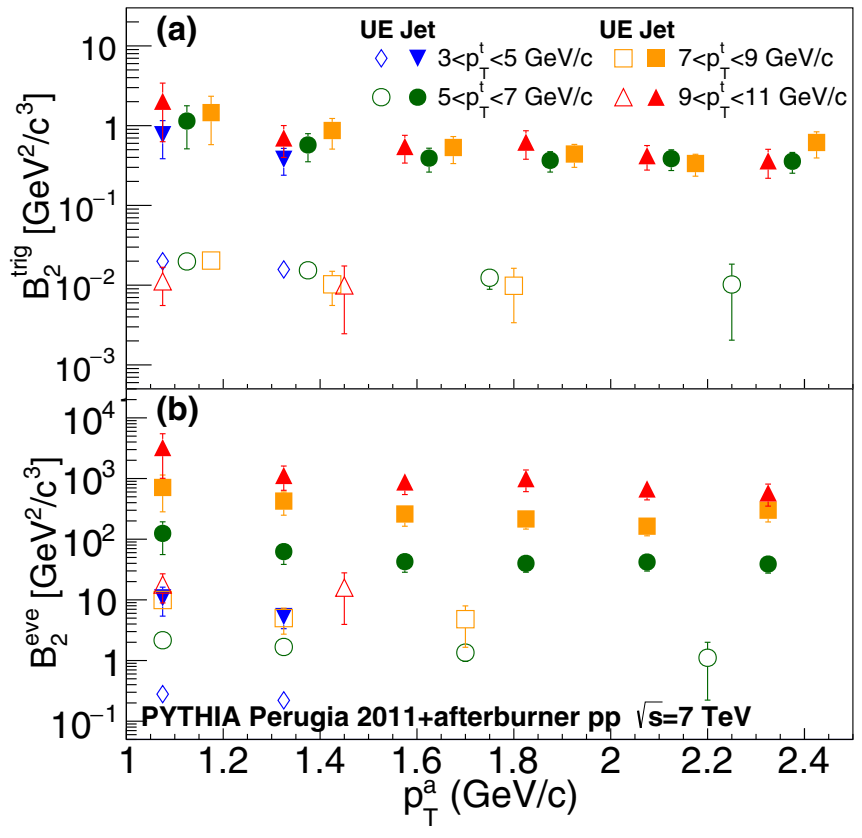

FIG. 7. The (a) $B_{2}^{\text {trig }}$ and (b) $B_{2}^{\text {eve }}$ calculated from the conditional yields for $3<p_{T}^{\mathrm{t}}<5 \mathrm{GeV} / c, 5<p_{T}^{\mathrm{t}}<7 \mathrm{GeV} / c, 7<p_{T}^{\mathrm{t}}<9$ $\mathrm{GeV} / c$, and $9<p_{T}^{\mathrm{t}}<11 \mathrm{GeV} / c$ for both the jetlike correlation and the underlying event (UE). Points have been displaced for visibility. The solid symbols denote the jetlike correlation while the open symbols represent UE.

where the superscript denotes the normalization by the number of triggers. An analogous quantity for normalization by the number of events can be calculated, $B_{2}^{\text {eve }}$, where $Y_{\text {trig }}$ is replaced by $Y_{\text {eve }}$.

Figure 7 shows the $B_{2}^{\text {trig }}$ and $B_{2}^{\text {eve }}$ calculated from the conditional yields for $3<p_{T}^{\mathrm{t}}<5 \mathrm{GeV} / c, 5<p_{T}^{\mathrm{t}}<7 \mathrm{GeV} / c$, $7<p_{T}^{\mathrm{t}}<9 \mathrm{GeV} / c$, and $9<p_{T}^{\mathrm{t}}<11 \mathrm{GeV} / c$ for the jetlike correlation and the underlying event. For both the jetlike correlation and the underlying event, $B_{2}^{\text {trig }}$ and $B_{2}^{\text {eve }}$ are roughly independent of $p_{T}^{a} / T$. In Fig. 7(a) the $B_{2}^{\text {trig }}$ for both the jetlike correlation and the underlying event are comparable for all trigger momenta. Since $B_{2}^{\text {trig }}$ is a measure of the particle composition of jets, this indicates that the deuteron to proton ratio is independent of the trigger momenta. Figure 7(b) shows $B_{2}^{\text {eve }}$, which increases with increasing $p_{T}^{\mathrm{t}}$. This shows that the relative contribution of a jet to inclusive $B_{2}$ increases with the jet momentum. The background for these correlations contain contributions not only from soft processes but also from hard processes unrelated to the trigger particle. At low momenta, several hard processes can occur per event. The increase in $B_{2}^{\text {eve }}$ may therefore occur from several hard processes in the event. It may also occur due to a higher overall multiplicity in events with a high momentum hadron.

Comparing Fig. 7 to Fig. 3 suggests that $B_{2}$ as a function of $p_{\mathrm{T}}$ in Fig. 3 may be dominated by contributions from the underlying event at lower $p_{\mathrm{T}}$ and for higher $p_{\mathrm{T}}$ the contribution from jetlike correlations dominates. This may suggest that deuteron production at high $p_{\mathrm{T}}$ is mostly due to jets. 


\section{CONCLUSIONS}

We modeled the production of deuterons through coalescence in $p+p$ collisions at $\sqrt{s}=7 \mathrm{TeV}$ by applying an afterburner, which coalesces protons and neutrons in PYTHIA. This model overpredicts the data, which is likely because PYTHIA uses the primary vertex as the origin of primary particles and therefore likely overestimates spatial correlations between protons and neutrons. The shapes of proton and deuteron spectra and hence $B_{2}$ as a function of $p_{\mathrm{T}}$ in the model are roughly consistent with the data. These calculations show that the rise in $B_{2}$ with momentum observed in data can indeed be generated by deuteron formation through coalescence in jets. We then showed that dihadron correlations can be used to separate deuterons in jets from those in the underlying event. The $d / p$ ratio calculated from the conditional spectra is higher in the jetlike correlation than in the underlying event. This may suggest an increase in deuteron production through coalescence in jets. If deuterons are produced through coalescence in jets, the conditional $B_{2}^{\text {trig }}$ should be roughly independent of the trigger particle momentum.

The use of dihadron correlations to separate deuterons in jets from those in the underlying event will allow deuteron production mechanisms to be studied in $p+p$ collisions. This approach may also be useful in heavy-ion collisions, where correlations between protons and neutrons can arise both through the production of jets and through hydrodynamical flow.

\section{ACKNOWLEDGMENTS}

We are grateful to Paul Stankus for useful comments in the manuscript. This work was supported in part by funding from the Division of Nuclear Physics of the US Department of Energy under Grant No. DE-FG02-96ER40982. N.S. acknowledges the support of DST-SERB Ramanujan Fellowship (D.O. No. SB/S2/RJN-084/2015). L.K. acknowledges the support of the SERB Grant No. ECR/2016/000109. This material is based upon work performed using computational resources within the University of Tennessee (UT) Advanced Computing Facility that are supported by the UT Joint Institute for Computational Sciences and partners. Any opinions, findings, and conclusions or recommendations expressed in this material are those of the authors and do not necessarily reflect the views of the University of Tennessee, the Joint Institute for Computational Sciences, or other ACF partners.
[1] L. Ahle et al. (E-802 Collaboration), Phys. Rev. C 60, 064901 (1999).

[2] T. A. Armstrong et al. (E-864 Collaboration), Phys. Rev. C 61, 064908 (2000).

[3] J. Barrette et al. (E-814 and E-877 Collaborations), Phys. Rev. C 61, 044906 (2000).

[4] S. Albergo et al., Phys. Rev. C 65, 034907 (2002).

[5] G. Ambrosini et al. (NA52 (NEWMASS) Collaboration), Phys. Lett. B 417, 202 (1998).

[6] I. G. Bearden et al., Eur. Phys. J. C 23, 237 (2002).

[7] S. V. Afanasev et al. (NA49 Collaboration), Phys. Lett. B 486, 22 (2000).

[8] T. Anticic et al. (NA49 Collaboration), Phys. Rev. C 69, 024902 (2004).

[9] T. Anticic et al. (NA49 Collaboration), Phys. Rev. C 85, 044913 (2012).

[10] T. Anticic et al. (NA49 Collaboration), Phys. Rev. C 94, 044906 (2016).

[11] S. Afanasiev et al. (PHENIX Collaboration), Phys. Rev. Lett. 99, 052301 (2007).

[12] B. I. Abelev et al. (STAR Collaboration), Phys. Rev. C 79, 034909 (2009).

[13] B. I. Abelev et al. (STAR Collaboration), arXiv:0909.0566.

[14] H. Agakishiev et al. (STAR Collaboration), Nature 473, 353 (2011) [Erratum: 475, 412 (2011)].

[15] J. Adam et al. (ALICE Collaboration), Phys. Rev. C 93, 024917 (2016).

[16] H. H. Gutbrod, A. Sandoval, P. J. Johansen, A. M. Poskanzer, J. Gosset, W. G. Meyer, G. D. Westfall, and R. Stock, Phys. Rev. Lett. 37, 667 (1976).

[17] S. T. Butler and C. A. Pearson, Phys. Rev. 129, 836 (1963).

[18] L. Adamczyk et al. (STAR Collaboration), Phys. Rev. C 94, 034908 (2016).
[19] J. I. Kapusta, Phys. Rev. C 21, 1301 (1980).

[20] S. Adler et al. (PHENIX Collaboration), Phys. Rev. Lett. 94, 122302 (2005).

[21] A. Adare et al. (PHENIX Collaboration), arXiv:1410.2559.

[22] R. Scheibl and U. W. Heinz, Phys. Rev. C 59, 1585 (1999).

[23] S. Acharya et al. (ALICE Collaboration), Phys. Rev. C 97, 024615 (2018).

[24] K. Blum, K. C. Y. Ng, R. Sato, and M. Takimoto, Phys. Rev. D 96, 103021 (2017).

[25] F. Donato, N. Fornengo, and P. Salati, Phys. Rev. D 62, 043003 (2000).

[26] E. Carlson, A. Coogan, T. Linden, S. Profumo, A. Ibarra, and S. Wild, Phys. Rev. D 89, 076005 (2014).

[27] N. Sharma (ALICE Collaboration), Nucl. Phys. A 956, 461 (2016).

[28] T. Sjostrand, S. Mrenna, and P. Z. Skands, J. High. Energy. Phys. 05 (2006) 026.

[29] L.-W. Chen, C. M. Ko, and B.-A. Li, Nucl. Phys. A 729, 809 (2003).

[30] P. Z. Skands, Phys. Rev. D 82, 074018 (2010).

[31] J. Adam et al. (ALICE Collaboration), Eur. Phys. J. C 75, 226 (2015).

[32] H.-d. Liu and Z. Xu, arXiv:nucl-ex/0610035.

[33] N. Sharma, J. Mazer, M. Stuart, and C. Nattrass, Phys. Rev. C 93, 044915 (2016).

[34] B. Abelev et al. (STAR Collaboration), Phys. Rev. C 80, 064912 (2009).

[35] B. Abelev et al. (STAR Collaboration), Phys. Lett. B 683, 123 (2010).

[36] G. Agakishiev et al. (STAR Collaboration), Phys. Rev. C 85, 014903 (2012). 